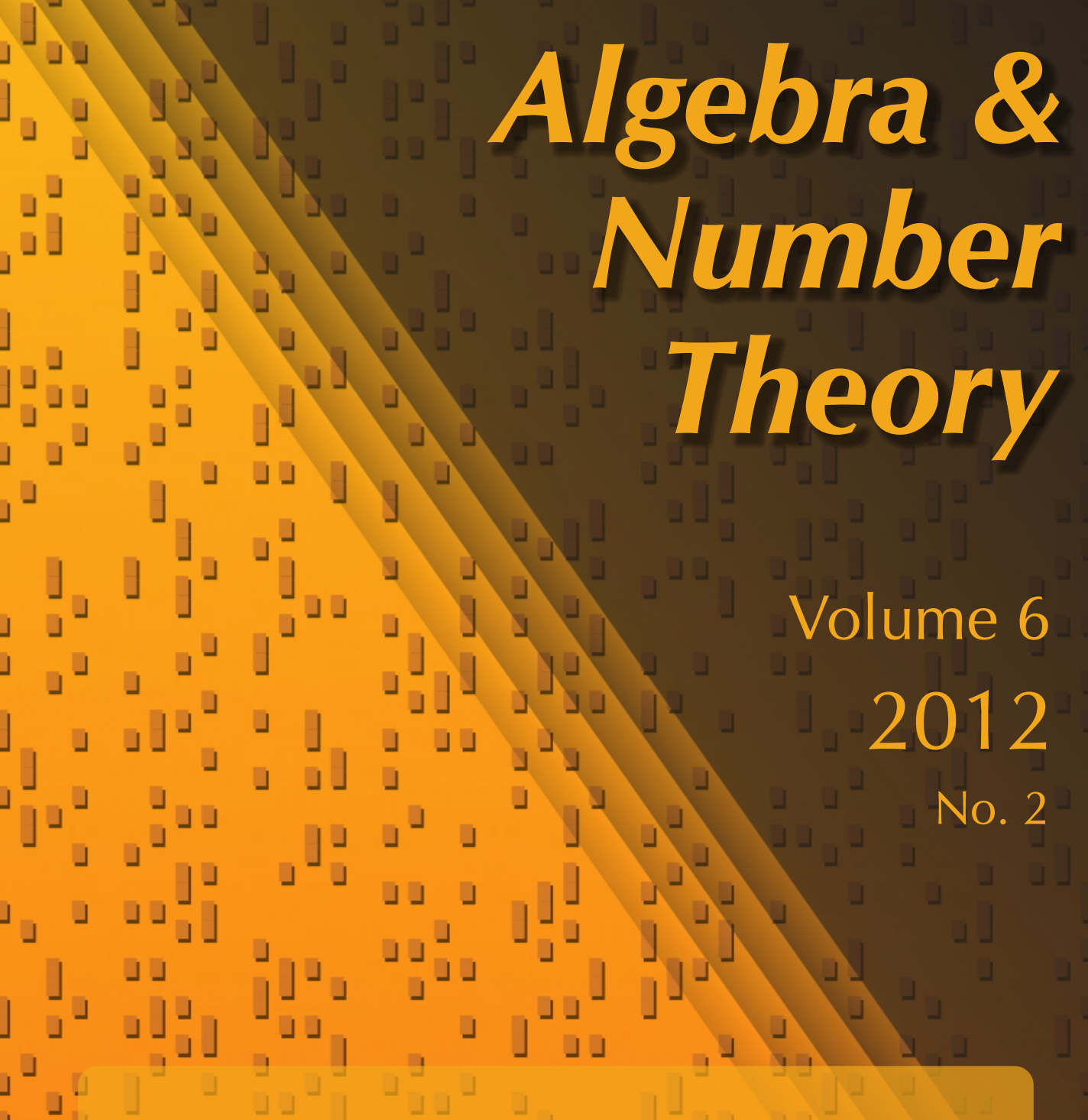

Fusion rules for abelian extensions of Hopf algebras

Christopher Goff

\lrcorner

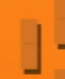

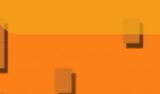

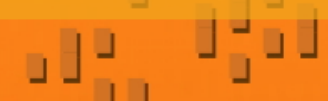

(נ)

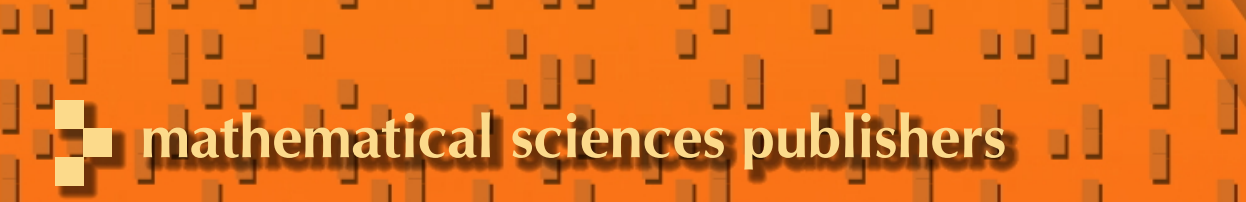




\title{
Fusion rules for abelian extensions of Hopf algebras
}

\author{
Christopher Goff
}

\begin{abstract}
We investigate the representation theory and fusion rules of a class of cocentral abelian (quasi-)Hopf extensions of Hopf algebras which includes twisted (generalized) quantum doubles of finite groups, and a certain quasi-Hopf algebra of Schauenburg associated to group-theoretical fusion categories. We then present a nontrivial example with noncommutative fusion rules.
\end{abstract}

\section{Introduction}

We present here a "ground-up" approach to attaining the fusion rules for a class of cocentral abelian extensions of Hopf algebras. Moreover, by not requiring strict coassociativity of the coproduct in the extension, our results are applicable not only to cocentral abelian (Hopf) extensions of Hopf algebras, but also to certain quasiHopf extensions as well. One such example, from [Schauenburg 2002], arises in the study of group-theoretical fusion categories (see also [Natale 2005]). (For a definition of group-theoretical fusion categories and basic properties, see [Etingof et al. 2005].) Another family of examples includes the twisted quantum double of a finite group, introduced in [Dijkgraaf et al. 1991], and the generalization which is defined in [Goff and Mason 2010].

In Section 2, we review definitions and notation, largely following [Kashina et al. 2002; Witherspoon 2004]. (For more information on extensions of Hopf algebras, consult [Andruskiewitsch 1996; Montgomery 1993], or, for quasi-Hopf extensions, [Masuoka 2002].) Then, Section 3 contains explicit formulas for irreducible characters and central idempotents for such extensions, as well as the inner product for which the irreducible characters form an orthonormal set. In Section 4, we write down the character of the tensor product representation and combine it with the inner product to deduce the fusion coefficients. The main result containing the fusion coefficients for irreducible representations, Theorem 4.5, is anticipated in [Witherspoon 2004] but is presented in this note without reference to Hochschild

MSC2000: primary 16S40, 18D10; secondary 16W30.

Keywords: fusion rules, Hopf algebras, quasi-Hopf, abelian extensions, group-theoretical fusion categories. 
cohomology per se. Corollary 4.6 points out the connection to the $K_{0}$-ring of a group-theoretical fusion category. Then, in Section 5, we apply these formulas to a generalized twisted quantum double of a finite group [Goff and Mason 2010]. Indeed, our Section 5 supersedes [Goff and Mason 2010, Section 3]. Finally, Section 6 contains a nontrivial example of a cocentral abelian extension having noncommutative fusion rules.

\section{Cocentral abelian extensions}

We follow closely the notation of [Kashina et al. 2002] with a few exceptions. First, our action is a right action, consistent with [Andruskiewitsch and Natale 2003]. Second, our modules will be right modules rather than left.

Let $L$ and $G$ be finite groups and let $\mathbb{F}$ be an algebraically closed field of characteristic not dividing $|G||L|$. An abelian extension $H$ is of the form

$$
0 \rightarrow(\mathbb{F} G)^{*} \rightarrow H \rightarrow \mathbb{F} L \rightarrow 0,
$$

where $H=(\mathbb{F} G)^{*} \#_{\sigma}^{\tau} \mathbb{F} L, \sigma: \mathbb{F} L \otimes \mathbb{F} L \rightarrow(\mathbb{F} G)^{*}$ is a group 2-cocycle, and $\tau$ : $(\mathbb{F} G)^{*} \rightarrow \mathbb{F} L \otimes \mathbb{F} L$ is the dual of a group 2-cocycle. The condition on $\mathbb{F}$ assures that $H$ is semisimple and cosemisimple. We specialize to a cocentral abelian extension, meaning that $(\mathbb{F} L)^{*} \subseteq Z\left(H^{*}\right)$, and thus that the coaction $\mathbb{F} L \rightarrow \mathbb{F} L \otimes(\mathbb{F} G)^{*}$ inherent in the extension is trivial. The cocentrality also has consequences for the tensor product structure on irreducible modules, as we will see in Section 4.

There is a right action of $\mathbb{F} L$ on $(\mathbb{F} G)^{*}$ which induces an action on $\mathbb{F} G$ via $\left(f\llcorner\ell)(g):=f\left(g\left\llcorner\ell^{-1}\right)\right.\right.$ for $g \in G, \ell \in L$, and extended linearly. Since $L$ acts as automorphisms of $(\mathbb{F} G)^{*}, L$ permutes the idempotents of the dual basis. Thus, the action can be viewed as an action of $L$ on $G$, also by automorphisms. For the basis $\left\{p_{g} \mid g \in G\right\}$, we have $p_{g}<\ell=p_{g}\left\llcorner\ell\right.$. Moreover, let $L_{g}$ be the stabilizer of $g$ in $L$ and $O(g)$ the orbit of $g$ under the action of $L$. That is,

$$
L_{g}=\{\ell \in L \mid g<\ell=g\} \text { and } \mathcal{O}(g)=\{g<\ell \mid \ell \in L\} .
$$

Let $T_{g}$ be a complete set of right coset representatives for $L_{g}$ in $L$. That is, $L=$ $\bigcup_{y \in T_{g}} L_{g} y$. Note that $\mathcal{O}(g)=\left\{g \leftarrow y \mid y \in T_{g}\right\}$.

We can write $\sigma$ and $\tau$ in terms of the dual basis via

$$
\sigma(x, y)=\sum_{g \in G} \sigma_{g}(x, y) p_{g} \quad \text { and } \quad \tau(x)=\sum_{g, h \in G} \tau_{g, h}(x)\left(p_{g} \otimes p_{h}\right),
$$

where $\sigma_{g}(x, y), \tau_{g, h}(x) \in \mathbb{F}$. There are many identities satisfied by $\sigma$ and $\tau$, such as

$$
\sigma_{g}\left\llcorner z(x, y) \sigma_{g}(z, x y)=\sigma_{g}(z, x) \sigma_{g}(z x, y)\right.
$$


and

$$
\tau_{g, h}(x) \tau_{g}{ }_{x, h}{ }_{x}(y) \sigma_{g}(x, y) \sigma_{h}(x, y)=\tau_{g, h}(x y) \sigma_{g h}(x, y),
$$

for all $g, h \in G, x, y, z \in L$.

Writing $p_{g} \# x$ as $p_{g} \bar{x}$, we can write multiplication in $H$ as

$$
p_{k} \bar{z} p_{h} \bar{y}=\delta_{k \leftarrow z, h} \sigma_{k}(z, y) p_{k} \overline{z y},
$$

for all $h, k \in G, y, z \in L$. We also occasionally write $p_{g}$ for $p_{g} \overline{1}$ and $\bar{x}$ for $\sum_{g} p_{g} \bar{x}$, whence $\bar{x} p_{g}=p_{g}\left\llcorner x^{-1} \bar{x}\right.$. The unit element is $\overline{1}$.

For a cocentral abelian extension, the comultiplication is

$$
\Delta\left(p_{g} \bar{x}\right)=\sum_{h \in G} \tau_{h, h^{-1} g}(x) p_{h} \bar{x} \otimes p_{h^{-1} g} \bar{x},
$$

for all $g \in G, x \in L$. The counit $\epsilon$ satisfies $\epsilon\left(p_{g} \bar{x}\right)=\delta_{g, 1}$. Finally, the antipode $S$ is given by

$$
S\left(p_{g} \bar{x}\right)=\sigma_{g^{-1}{ }_{x}}\left(x^{-1}, x\right)^{-1} \tau_{g^{-1}, g}(x)^{-1} p_{g^{-1}{ }_{x}} \overline{x^{-1}} .
$$

Remark 2.1. For $H$ to be a Hopf algebra, $\Delta$ must be coassociative, which implies a certain condition on $\tau$. We require only quasicoassociativity, which implies the existence of other structures, and a related condition on $\tau$. We omit these details here, as all of our examples are proved elsewhere [Dijkgraaf et al. 1991; Natale 2005; Andruskiewitsch 1996] to be either coassociative or quasicoassociative.

\section{Modules and characters}

Irreducible modules for $H$ are induced from irreducible modules for the group algebra of $L_{g}$, but twisted by the 2-cocycle $\sigma_{g}$. Select one $g$ from each orbit under the action of $L$, then select $T_{g}$, a set of right coset representatives. Let

$$
H_{g}:=(\mathbb{F} G)^{*} \#_{\sigma} \mathbb{F} L_{g}
$$

If $V$ is a right projective $\sigma_{g}$-representation space for $L_{g}$, then $V \otimes p_{g}$ is a right $H_{g}$-module via

$$
\left(v \otimes p_{g}\right) \cdot\left(p_{h} \bar{x}\right)=\delta_{g, h}\left(v \cdot x \otimes p_{g}\right)
$$

for all $v \in V, h \in G, x \in L_{g}$.

The irreducible modules for $H$ are induced from these. Let $\widehat{V}=\left(V \otimes p_{g}\right) \otimes_{H_{g}} H$, which is then a right $H$-module under right multiplication by $H$. In other words,

$$
\widehat{V}=\sum_{y \in T_{g}}\left(v \otimes p_{g}\right) \otimes \bar{y}
$$


with action given by

$$
\begin{aligned}
{\left[\left(v \otimes p_{g}\right) \otimes \bar{y}\right] \cdot p_{h} \bar{x} } & =\left(v \otimes p_{g}\right) \sigma_{h\left\llcorner y^{-1}\right.}(y, x) p_{h\left\llcorner y^{-1}\right.} \overline{y x} \\
& =\left(v \otimes p_{g}\right) \delta_{g, h\left\llcorner y^{-1}\right.} \sigma_{g}(y, x) p_{g} \overline{w y^{\prime}} \\
& =\left(v \otimes p_{g}\right) \delta_{g, h<y^{-1}} \sigma_{g}(y, x) \sigma_{g}\left(w, y^{\prime}\right)^{-1}\left(p_{g} \bar{w}\right)\left(\overline{y^{\prime}}\right) \\
& =\delta_{g, h\left\llcorner y^{-1}\right.} \frac{\sigma_{g}(y, x)}{\sigma_{g}\left(w, y^{\prime}\right)}\left[\left(v \cdot w \otimes p_{g}\right) \otimes \overline{y^{\prime}}\right]
\end{aligned}
$$

where $w \in L_{g}, y^{\prime} \in T_{g}$ are chosen so that $w y^{\prime}=y x$.

We introduce the notation $V_{(g, \varphi)}$ to represent the $H$-module induced from the projective $\sigma_{g}$-representation of $L_{g}$ that has character $\varphi$, and we let $\rho_{(g, \varphi)}$ be the representation of $V_{(g, \varphi)}$, and $\chi_{(g, \varphi)}$ its character. Then one calculates

$$
\chi_{(g, \varphi)}\left(p_{h} \bar{x}\right)=\delta_{g}\left\llcorner y, h \delta_{y x y^{-1} \in L_{g}} \frac{\sigma_{g}(y, x)}{\sigma_{g}\left(y x y^{-1}, y\right)} \varphi\left(y x y^{-1}\right),\right.
$$

where $y$ is the unique element of $T_{g}$ that maps $g$ to $h$. We reiterate that $V_{(g, \varphi)}$ is irreducible if and only if $\varphi$ is.

Remark 3.1. This can be seen as

$$
\chi_{(g, \varphi)}\left(p_{h} \bar{x}\right)=\delta_{g\llcorner y, h} \delta_{x \in L_{h}} \varphi^{(y)}(x),
$$

where $\varphi^{(y)}$ is a projective representation of $L_{h}=L_{g}^{y}$ (conjugate to $\varphi$ ) with cocycle $\sigma_{g}\left\llcorner y=\sigma_{h}\right.$. See [Costache 2009, Lemma 59] for a similar calculation.

Before writing down the central idempotents, we first note that the character $\chi_{\text {reg }}$ of the regular representation $\rho_{\text {reg }}$ on $H$ satisfies $\chi_{\text {reg }}\left(p_{h} \bar{x}\right)=\delta_{x, 1}|\mathcal{O}(h)|\left|L_{h}\right|=$ $\delta_{x, 1}|L|$, and that, from the semisimplicity of $H$,

$$
\rho_{\text {reg }}=\bigoplus_{(h, \psi)} \chi_{(h, \psi)}\left(1_{H}\right) \rho_{(h, \psi)},
$$

where $h$ ranges over the orbits and $\psi$ ranges over the irreducible projective $\sigma_{h^{-}}$ representations of $L_{h}$. Let $z_{(g, \varphi)}$ denote the central idempotent corresponding to the representation $\rho_{(g, \varphi)}$. Then $\rho_{(h, \psi)}\left(z_{(g, \varphi)}\right)=\delta_{g, h} \delta_{\varphi, \psi}(\operatorname{dim} \varphi)\left|L: L_{g}\right|$ id.

Set $z_{(g, \varphi)}=\sum_{c \in G, d \in L} \alpha_{c, d} p_{c} \bar{d}$. We find the $\alpha_{c, d}$ by determining the value of the regular character on $S\left(p_{a^{-1}} \bar{b}\right) z_{(g, \varphi)}$ two ways. First,

$$
\begin{aligned}
\chi_{\mathrm{reg}}\left(S\left(p_{a^{-1}} \bar{b}\right) z_{(g, \varphi)}\right) & =\sum_{c \in G, d \in L} \sigma_{a\llcorner b}\left(b^{-1}, b\right)^{-1} \tau_{a, a^{-1}}(b)^{-1} \alpha_{c, d} \chi_{\mathrm{reg}}\left(p_{a\llcorner b} \overline{b^{-1}} p_{c} \bar{d}\right) \\
& =\tau_{a, a^{-1}}(b)^{-1} \alpha_{a, b}|L| .
\end{aligned}
$$

On the other hand, we have

$$
\rho_{\text {reg }}\left(S\left(p_{a^{-1}} \bar{b}\right) z_{(g, \varphi)}\right)=(\operatorname{dim} \varphi)\left|L: L_{g}\right| \rho_{(g, \varphi)}\left(S\left(p_{a^{-1}} \bar{b}\right)\right),
$$


which means

$\chi_{\text {reg }}\left(S\left(p_{a^{-1}} \bar{b}\right) z_{(g, \varphi)}\right)=(\operatorname{dim} \varphi)\left|L: L_{g}\right| \sigma_{a\llcorner b}\left(b^{-1}, b\right)^{-1} \tau_{a, a^{-1}}(b)^{-1} \chi_{(g, \varphi)}\left(p_{a\llcorner b} \overline{b^{-1}}\right)$

Solving for $\alpha_{a, b}$, we obtain

$$
z_{(g, \varphi)}=\frac{(\operatorname{dim} \varphi)}{\left|L_{g}\right|} \sum_{a \in G, b \in L} \frac{1}{\sigma_{a\llcorner b}\left(b^{-1}, b\right)} \chi_{(g, \varphi)}\left(p_{a\llcorner b} \overline{b^{-1}}\right)\left(p_{a} \bar{b}\right) .
$$

Simplifying somewhat using the delta functions within $\chi_{(g, \varphi)}$, we have:

Lemma 3.2. The central idempotent of $H$ corresponding to $V_{(g, \varphi)}$ is

$$
z_{(g, \varphi)}=\frac{(\operatorname{dim} \varphi)}{\left|L_{g}\right|} \sum_{a \in G} \sum_{b \in L_{a}} \frac{1}{\sigma_{a}\left(b^{-1}, b\right)} \chi_{(g, \varphi)}\left(p_{a} \overline{b^{-1}}\right)\left(p_{a} \bar{b}\right) .
$$

Note that the first sum could be over $a \in \mathbb{O}(g)$, as $\chi=0$ otherwise.

\section{Proposition 3.3. Letting}

$$
\langle\alpha, \beta\rangle=\frac{1}{|L|} \sum_{a \in G} \sum_{b \in L_{a}} \frac{1}{\sigma_{a}\left(b^{-1}, b\right)} \alpha\left(p_{a} \overline{b^{-1}}\right) \beta\left(p_{a} \bar{b}\right),
$$

where $\alpha, \beta$ are characters of $H$, defines an inner product on the space of characters of $H$. The irreducible characters form an orthonormal basis with respect to this inner product.

We give three proofs to demonstrate the consistency with the character theory of projective representations of finite groups, and to demonstrate the relationship between certain conjugates of projective representations.

First proof. Clearly, (5) is linear in each component. The symmetry of (5) follows from (1) because $b \in L_{a}$. Using Lemma 3.2, we have

$$
\begin{aligned}
\left\langle\chi_{(g, \varphi)}, \chi_{(h, \psi)}\right\rangle & =\frac{1}{|L|} \sum_{a \in G} \sum_{b \in L_{a}} \frac{1}{\sigma_{a}\left(b^{-1}, b\right)} \chi_{(g, \varphi)}\left(p_{a} \overline{b^{-1}}\right) \chi_{(h, \psi)}\left(p_{a} \bar{b}\right) \\
& =\frac{1}{|L|} \chi_{(h, \psi)}\left(\frac{\left|L_{g}\right|}{\operatorname{dim} \varphi} z_{(g, \varphi)}\right) \\
& =\left(\frac{1}{|L|} \frac{\left|L_{g}\right|}{\operatorname{dim} \varphi}\right)(\operatorname{dim} \varphi)\left|L: L_{g}\right| \cdot \delta_{g, h} \delta_{\varphi, \psi}=\delta_{g, h} \delta_{\varphi, \psi} .
\end{aligned}
$$

Second proof. From (3), we obtain that $a \in \mathcal{O}(g) \cap \mathcal{O}(h)$ and thus $g=h$ or else the inner product is zero. Thus 


$$
\begin{aligned}
& \langle\chi(g, \varphi), \chi(h, \psi)\rangle \\
& =\frac{\delta_{g, h}}{|L|} \sum_{\substack{a \in \mathcal{O}(g) \\
[a=g \leftarrow y]}} \sum_{b \in L_{a}} \frac{\sigma_{g}\left(y, b^{-1}\right) \sigma_{g}(y, b)}{\sigma_{a}\left(b^{-1}, b\right) \sigma_{g}\left(y b^{-1} y^{-1}, y\right) \sigma_{g}\left(y b y^{-1}, y\right)} \varphi\left(y b^{-1} y^{-1}\right) \psi\left(y b y^{-1}\right) \\
& =\frac{\delta_{g, h}}{|L|} \sum_{\substack{a \in \mathcal{O}(g) \\
[a=g\llcorner y]}} \sum_{b \in L_{a}} \frac{1}{\sigma_{g}\left(y b^{-1} y^{-1}, y b y^{-1}\right)} \varphi\left(y b^{-1} y^{-1}\right) \psi\left(y b y^{-1}\right)
\end{aligned}
$$

by repeated application of (1). Hence

$$
\left\langle\chi_{(g, \varphi)}, \chi_{(h, \psi)}\right\rangle=\frac{\delta_{g, h}}{\left|L_{g}\right|} \sum_{c \in L_{g}} \frac{1}{\sigma_{g}\left(c^{-1}, c\right)} \varphi\left(c^{-1}\right) \psi(c)=\delta_{g, h}\langle\varphi, \psi\rangle_{L_{g}}=\delta_{g, h} \delta_{\varphi, \psi}
$$

Here, $\langle\cdot, \cdot\rangle_{L_{g}}$ denotes the usual inner product for projective $\sigma_{g}$-representations of $L_{g}$. See [Nauwelaerts and Van Oystaeyen 1991, Proposition 2.8], for instance.

Third proof. Using Remark 3.1,

$$
\begin{aligned}
\left\langle\chi_{(g, \varphi)}, \chi_{(h, \psi)}\right\rangle & =\frac{\delta_{g, h}}{|L|} \sum_{\substack{a \in \mathcal{O}(g) \\
[a=g-y]}} \sum_{b \in L_{a}} \frac{1}{\sigma_{a}\left(b^{-1}, b\right)} \varphi^{(y)}\left(b^{-1}\right) \psi^{(y)}(b) \\
& =\frac{\delta_{g, h}}{|L|} \sum_{a \in \mathcal{O}(g)}\left|L_{a}\right|\left\langle\varphi^{(y)}, \psi^{(y)}\right\rangle_{L_{a}}=\delta_{g, h} \delta_{\varphi, \psi} .
\end{aligned}
$$

It is clear that $\varphi^{(y)}=\psi^{(y)}$ if and only if $\varphi=\psi$.

\section{Fusion rules}

The character of the tensor product representation (via $\Delta$ ) is

$$
\begin{aligned}
& \chi_{(g, \varphi) \otimes(h, \psi)}\left(p_{a} \bar{b}\right) \\
& =\quad \sum_{f \in G} \quad \delta_{b \in L_{f} \cap L_{f^{-1} a}} \varphi\left(y b y^{-1}\right) \psi\left(w b w^{-1}\right) \frac{\tau_{f, f^{-1} a}(b) \sigma_{g}(y, b) \sigma_{g}(w, b)}{\sigma_{g}\left(y b y^{-1}, y\right) \sigma_{g}\left(w b w^{-1}, b\right)} \\
& {\left[f \in \mathscr{O}(g), f^{-1} a \in \mathscr{O}(h)\right]} \\
& \text { [ } f=g\left\llcorner y, f^{-1} a=h\llcorner w]\right. \\
& =\quad \sum_{f \in G} \quad \delta_{b \in L_{f} \cap L_{f^{-1} a}} \tau_{f, f^{-1} a}(b) \varphi^{(y)}(b) \psi^{(w)}(b) \\
& {\left[f \in \mathscr{O}(g), f^{-1} a \in \mathscr{O}(h)\right]} \\
& {\left[f=g\left\llcorner y, f^{-1} a=h\llcorner w]\right.\right.} \\
& =\quad \sum_{f \in G} \quad \delta_{b \in L_{f} \cap L_{f^{-1} a}}\left[\varphi^{(y)} \otimes \psi^{(w)} \tau_{f, f^{-1} a}\right](b) \text {, } \\
& {\left[f \in \mathscr{O}(g), f^{-1} a \in \mathbb{O}(h)\right]} \\
& \text { [ } f=g\left\llcorner y, f^{-1} a=h\llcorner w \text { ] }\right.
\end{aligned}
$$


where $\left[\varphi^{(y)} \otimes \psi^{(w)} \tau_{f, f^{-1} a}\right]$ is a projective representation (of $L_{f} \cap L_{f^{-1} a} \leq L_{a}$ ) with cocycle $\sigma_{a}$. As explained in [Witherspoon 2004, (4.7)], the cocentrality of the extension, and the fact that the coproduct $\Delta$ is an algebra map, together imply that $\sigma_{a}$ is cohomologous to $\sigma_{f} \cdot \sigma_{f^{-1} a}$ on $L_{f} \cap L_{f^{-1} a}$ via $\tau_{f, f^{-1} a}$. This is the content of Equation (2), which depends on the assumption of cocentrality.

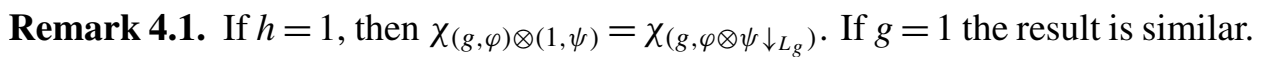
Hence, the irreducible representations induced from $1 \in G$ are in the center of the fusion algebra and their tensor products with other modules can be reduced to a calculation in the appropriate stabilizer. This generalizes a similar result in [Goff and Mason 2010].

We need two lemmas before calculating the fusion coefficients.

Lemma 4.2. Let $a, f \in G, y \in L$.

(1) Let $\alpha$ and $\beta$ be projective $\sigma_{f}$-representations of $L_{f}$. Then

$$
\langle\alpha, \beta\rangle_{L_{f}}=\left\langle\alpha^{(y)}, \beta^{(y)}\right\rangle_{L_{f}^{y}}
$$

Note that $\alpha^{(y)}$ and $\beta^{(y)}$ are $\sigma_{f}-y$-representations of $L_{f}\left\llcorner y=L_{f}^{y}\right.$.

(2) Let $\alpha$ be a $\sigma_{f}$-representation of $L_{f}$ and let $\beta$ be a $\sigma_{f^{-1}}$-representation of $L_{f^{-1} a}$. Then

$$
\left[\alpha \otimes \beta \tau_{f, f^{-1} a}\right]^{(y)}=\left[\alpha^{(y)} \otimes \beta^{(y)} \tau_{f<y, f^{-1} a<y}\right]
$$

as $\sigma_{a}<y$-representations of $L_{f}^{y} \cap L_{f^{-1} a}^{y} \leq L_{a}^{y}$.

Proof. The proof is straightforward, using (4), (1), and (2).

We need a way to calculate products of $L$-orbits in $\mathbb{C} G$. The following formula appears in [Witherspoon 2004, Proof of Theorem 4.8], where the author relies on standard trace map properties of the $L$-algebra $\mathbb{Z} G$, citing general results of [Thévenaz 1995]. Our proof is specific to group actions on sets. Recall that if $L$ acts on $G$, then $L$ also acts on $G \times G$ diagonally: $\left(g_{1}, g_{2}\right)<\ell=\left(g_{1}<\ell, g_{2}<\ell\right)$ for $\ell \in L, g_{1}, g_{2} \in G$.

Lemma 4.3. Let $g, h \in G$. Then

$$
\mathcal{O}(g) \mathcal{O}(h)=\sum_{x \in D} \mid L_{(g\llcorner x) h}: L_{g}\left\llcorner x \cap L_{h} \mid \mathcal{O}((g\llcorner x) h),\right.
$$

where $D$ is a complete set of $L_{g} \backslash L / L_{h}$ double coset representatives.

Proof. Consider the orbits of the diagonal action of $L$ on $G \times G$. Evidently, $y \in L_{g} x L_{h}$ if and only if $\mathrm{O}_{L}((g \leftarrow x, h))=\mathrm{O}_{L}((g<y, h))$. Now pick $x \in D$ and consider the image of $O_{L}((g<x, h))$ in $G$ under the product map. Clearly, the product $(g \leftarrow x) h$ is fixed by $L_{(g\llcorner x) h}$ but also each component is fixed by 
$L_{g}^{x} \cap L_{h} \leq L_{(g\llcorner x) h}$. So, the number of distinct ordered pairs $(g<x w, h<w)$ such that $(g \leftarrow x w)(h \leftarrow w)=(g \leftarrow x) h$ is $\left|L_{(g\llcorner x) h}: L_{g}^{x} \cap L_{h}\right|$. Since $L$ acts by

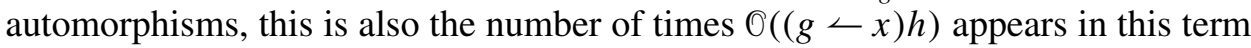
of the sum.

Remark 4.4. The right hand side in Lemma 4.3 cannot generally be interpreted as a summation over distinct orbits. There may be $y \notin L_{g} x L_{h}$ for which $\mathbb{O}((g \leftarrow$

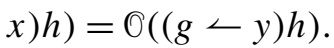

Anticipated in [Witherspoon 2004, Theorem 4.8], the following theorem gives the fusion coefficients for irreducible representations of $H$.

Theorem 4.5. Let $g, h, k \in G$ and let $\varphi$ be a $\sigma_{g}$-representation of $L_{g}$, $\psi$ a $\sigma_{h^{-}}$ representation of $L_{h}$, and $\gamma$ a $\sigma_{k}$-representation of $L_{k}$ and consider the corresponding induced modules of $H$. Then

$$
\left\langle\chi_{(k, \gamma)}, \chi_{(g, \varphi) \otimes(h, \psi)}\right\rangle=\sum_{\substack{x \in D \\\left(g \llcorner x ) h \in \mathbb { O } ( k ) \\ \left(g\left\llcorner x w^{\prime}\right)\left(h\left\llcorner w^{\prime}\right)=k\right]\right.\right.}}\left\langle\gamma,\left[\varphi^{\left(x w^{\prime}\right)} \otimes \psi^{\left(w^{\prime}\right)} \tau_{g}\left\llcorner x w^{\prime}, h\left\llcorner w^{\prime}\right]\right\rangle_{L_{g}^{x w^{\prime}} \cap L_{h}^{w^{\prime}}}\right.\right.
$$

where $D$ is a set of those $L_{g} \backslash L / L_{h}$ double coset representatives $x$ satisfying

$$
(g<x) h \in \mathcal{O}(k),
$$

and the inner product on $L_{g}^{x w^{\prime}} \cap L_{h}^{w^{\prime}} \leq L_{k}$ is of projective $\sigma_{k}$-representations.

Proof. Using the inner product (5), we have

$$
\begin{aligned}
& \left\langle\chi_{(k, \gamma)}, \chi_{(g, \varphi) \otimes(h, \psi)}\right\rangle \\
& \begin{aligned}
&=\frac{1}{|L|} \sum_{\substack{a \in \mathscr{O}(k) \\
[a=k<-z]}} \sum_{\substack{f \in \mathcal{O}(g) \\
f^{-1} a \in \mathscr{O}(h) \\
\left[f=g\llcorner y] \\
\left[f^{-1} a=h\llcorner-w]\right.\right.}} \sum_{b \in L_{a} \cap L_{f}} \gamma\left(z b^{-1} z^{-1}\right) \varphi\left(y b y^{-1}\right) \psi\left(w b w^{-1}\right) \\
& \cdot \frac{\tau_{f, f^{-1} a}(b) \sigma_{k}\left(z, b^{-1}\right) \sigma_{g}(y, b) \sigma_{h}(w, b)}{\sigma_{a}\left(b^{-1}, b\right) \sigma_{k}\left(z b^{-1} z^{-1}, z\right) \sigma_{g}\left(y b y^{-1}, y\right) \sigma_{h}\left(w b w^{-1}, w\right)}
\end{aligned} \\
& =\frac{1}{|L|} \sum_{\substack{a \in \mathcal{O}(k) \\
[a=k<z]}} \sum_{\substack{f \in \mathscr{O}(g) \\
f^{-1} a \in \mathscr{O}(h) \\
\left[f=g\llcorner y] \\
\left[f^{-1} a=h\llcorner w]\right.\right.}} \sum_{b \in L_{a} \cap L_{f}} \frac{\tau_{f, f^{-1} a}(b)}{\sigma_{a}\left(b^{-1}, b\right)} \gamma^{(z)}\left(b^{-1}\right) \varphi^{(y)}(b) \psi^{(w)}(b) \\
& =\frac{1}{|L|} \sum_{\substack{a \in \mathscr{O}(k) \\
[a=k<z]}} \sum_{\substack{\left.f \in \mathscr{O}(g) \\
f^{-1} a \in \mathbb{O}(h) \\
f=g-y\right] \\
\left[f^{-1} a=h\llcorner w]\right.}}\left|L_{f} \cap L_{f^{-1} a}\right|\left\langle\gamma^{(z)},\left[\varphi^{(y)} \otimes \psi^{(w)} \tau_{f, f^{-1} a}\right]\right\rangle_{L_{f} \cap L_{f^{-1} a}} .
\end{aligned}
$$


By Lemma 4.2 this is equal to

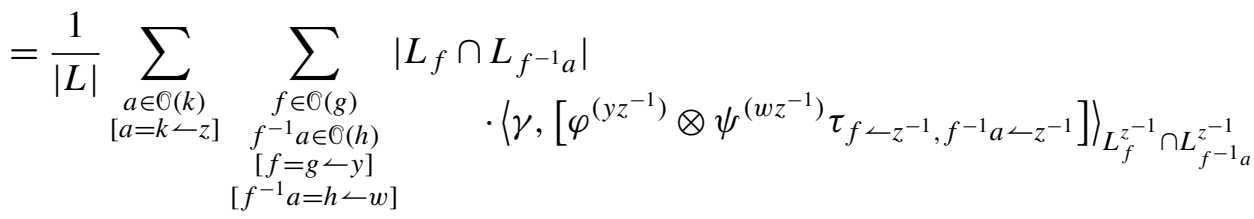

$$
\begin{aligned}
& =\frac{1}{\left|L_{k}\right|} \sum_{\substack{f \in \mathcal{O}(g) \\
f^{-1} k \in \mathcal{O}(h) \\
\left[f=g-y^{\prime}\right] \\
\left[f^{-1} k=h L^{\prime}\right]}}\left|L_{f} \cap L_{f^{-1} k}\right|\left\langle\gamma,\left[\varphi^{\left(y^{\prime}\right)} \otimes \psi^{\left(w^{\prime}\right)} \tau_{f, f^{-1} k}\right]\right\rangle_{L_{f} \cap L_{f^{-1} k}},
\end{aligned}
$$

and by Lemma 4.3 this can further be written as

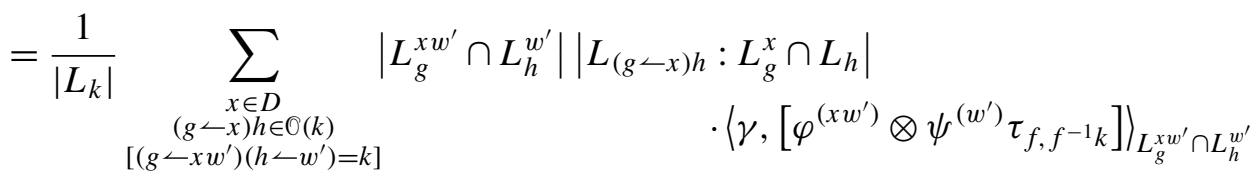

$$
\begin{aligned}
& =\sum_{\substack{x \in D \\
\left(g \llcorner x ) h \in \mathbb { O } ( k ) \\
\left[\left(g\left\llcorner x w^{\prime}\right)\left(h\left\llcorner w^{\prime}\right)=k\right]\right.\right.\right.}}\left\langle\gamma,\left[\varphi^{\left(x w^{\prime}\right)} \otimes \psi^{\left(w^{\prime}\right)} \tau_{g}\left\llcorner x w^{\prime}, h\left\llcorner w^{\prime}\right]\right\rangle_{L_{g}^{x w^{\prime}} \cap L_{h}^{w^{\prime}}},\right.\right.
\end{aligned}
$$

where $D$ is a set of $L_{g} \backslash L / L_{h}$ double coset representatives with $(g<x) h \in \mathcal{O}(k)$. Thus, the fusion rules for $H$ modules can be determined from the fusion rules for projective $\sigma_{k}$-representations restricted to certain subgroups of $L_{k}$.

As stated before, the theorem holds for certain quasi-Hopf extensions, including the examples in the following corollary and the next section.

Corollary 4.6. The fusion rules in Theorem 4.5 describe the $K_{0}$-ring for the grouptheoretical module category $\mathscr{b}(G \rtimes L, \omega, L, 1)$, where $\omega \in H^{3}\left(G \rtimes L, \mathbb{F}^{*}\right)$ is the 3cocycle associated to $[\sigma, \tau]$ in the relevant Kac exact sequence. See [Schauenburg 2002; Natale 2003; Masuoka 2002] for further cohomological details.

Proof. Indeed, the theorem holds whenever the structure maps and (1) and (2) hold, even if $H$ is a quasi-Hopf algebra (with coassociator $\Phi$ ), because the fusion rules for $H$ do not depend on the associativity constraint (determined by $\Phi$ ) in the category of right $H$-modules, Mod- $H$. Thus these fusion rules hold for a certain quasiHopf algebra of Schauenburg, denoted $\left(A^{\mathrm{op}}, \Phi\right)$ by Natale [2005], in the case when $A=(\mathbb{F} G)^{*} \#_{\sigma}^{\tau} \mathbb{F} L$, and the left action $\triangleright$ of $G$ on $L$ is trivial; i.e., when $G L=G \rtimes L$. (In this case, the structure maps and cocycles are exactly as in Section 2.) Natale, in the proof of her Theorem 4.4, cites [Schauenburg 2002] to demonstrate that ( $\left.A^{\text {op }}, \Phi\right)$-Mod is tensor-equivalent to $\mathscr{C}(G \rtimes L, \omega, L, 1)$, where $\omega \in H^{3}\left(G \rtimes L, \mathbb{F}^{*}\right)$ is the 3 -cocycle associated to $[\sigma, \tau]$ in the Kac exact sequence. 


\section{Example: generalized twisted quantum doubles of finite groups}

Other examples of abelian extensions satisfying the structure maps of Section 2 (and hence having fusion rules determined by Theorem 4.5) include twisted quantum doubles of finite groups [Dijkgraaf et al. 1991] and generalized twisted doubles of finite groups [Goff and Mason 2010]. We expand on the latter, but using right modules here. As mentioned earlier, this section supersedes [Goff and Mason 2010, Section 3].

Let $G$ be a finite group, $N$ a normal subgroup, and $\bar{G}:=G / N$. We use the bar notation for elements in $\bar{G}$, i.e., if $g \in G$ then $\bar{g}=g N \in \bar{G}$. Then $G$ acts naturally on $\bar{G}$ via conjugation, namely $\bar{g} \leftarrow x:=x^{-1} \bar{g} x=\bar{g}^{x}=\bar{g}^{x}=\bar{x}^{-1} \bar{g} \bar{x}$, for all $x \in G, \bar{g} \in \bar{G}$.

In addition, let $\omega \in H^{3}\left(\bar{G}, \mathbb{F}^{*}\right)$, and let $\omega^{\prime}:=\operatorname{Infl}_{\bar{G}}^{G} \omega$. In analogy with $\sigma$ and $\tau$, define $\theta: \mathbb{F} G \otimes \mathbb{F} G \rightarrow \mathbb{F} \bar{G}^{*}$ and $\gamma: \mathbb{F} \bar{G}^{*} \rightarrow \mathbb{F} G \otimes \mathbb{F} G$ via

$$
\theta=\sum_{\bar{g} \in \bar{G}} \theta_{\bar{g}} \text { and } \gamma=\sum_{x, y \in \bar{G}} \gamma_{0}(x, y),
$$

where

$$
\theta_{\bar{g}}(x, y)=\frac{\omega(\bar{g}, \bar{x}, \bar{y}) \omega\left(\bar{x}, \bar{y}, \bar{g}^{x y}\right)}{\omega\left(\bar{x}, \bar{g}^{x}, \bar{y}\right)}, \quad \gamma_{\bar{g}}(x, y)=\frac{\omega(\bar{x}, \bar{y}, \bar{g}) \omega\left(\bar{g}, \bar{x}^{g}, \bar{y}^{g}\right)}{\omega\left(\bar{x}, \bar{g}, \bar{y}^{g}\right)} .
$$

Notice that $\theta_{\bar{g}}$ and $\gamma_{\bar{g}}$ could be thought of as functions from $\mathbb{F} \bar{G} \otimes \mathbb{F} \bar{G}$ to $\mathbb{F}^{*}$ since they pass to the quotient $\bar{G}$. The generalized twisted double is then $D^{\omega}(G, \bar{G})=$ $(\mathbb{F} \bar{G})^{*} \#_{\theta}^{\gamma}(\mathbb{F} G)$. The maps $\theta$ and $\gamma$ satisfy (1) and (2), mutatis mutandis [Dijkgraaf et al. 1991].

The irreducible (right) modules of $D^{\omega}(G, \bar{G})$ are induced from irreducible projective representations of centralizers. In particular, the character of the irreducible projective $\theta_{\bar{g}}$-representation $\varphi$ of $C_{G}(\bar{g})$ is given by

$$
\begin{aligned}
\widehat{\chi}_{(\bar{g}, \varphi)}(e(\bar{h}) \bowtie x) & =\delta_{\bar{g}^{y}, \bar{h}} \delta_{y x y^{-1} \in C_{G}(\bar{g})} \frac{\theta_{\bar{g}}(y, x)}{\theta_{\bar{g}}\left(y x y^{-1}, y\right)} \varphi\left(y x y^{-1}\right) \\
& =\delta_{\bar{g}^{y}, \bar{h}} \delta_{x \in C_{G}(\bar{h})} \varphi^{(y)}(x) .
\end{aligned}
$$

Consistent with (5), the inner product on characters is given by

$$
\langle\alpha, \beta\rangle=\frac{1}{|G|} \sum_{\bar{k} \in \bar{G}} \sum_{x \in C_{G}(\bar{k})} \frac{1}{\theta_{\bar{k}}\left(\bar{x}^{-1}, \bar{x}\right)} \alpha\left(e(\bar{k}) \bowtie x^{-1}\right) \beta(e(\bar{k}) \bowtie x),
$$

and thus the fusion coefficients are given by

$$
\left\langle\widehat{\chi}_{(\bar{k}, \lambda)}, \widehat{\chi}_{(\bar{g}, \varphi) \otimes(\bar{h}, \psi)}\right\rangle=\sum_{\substack{x \in D \\\left[\bar{g}^{x w^{\prime}} \bar{h}^{w^{\prime}}=\bar{k}\right]}}\left\langle\lambda,\left[\varphi^{\left(x w^{\prime}\right)} \otimes \psi^{\left(w^{\prime}\right)} \gamma_{O}\left(\bar{g}^{x w^{\prime}}, \bar{h}^{w^{\prime}}\right)\right]\right\rangle_{C_{G}\left(\bar{g}^{x w^{\prime}}\right) \cap C_{G}\left(\bar{h}^{w^{\prime}}\right)}
$$


where $D$ is a set of $C_{G}(\bar{g}) \backslash G / C_{G}(\bar{h})$ double coset representatives with $\bar{g}^{x} \bar{h} \in \mathcal{O}(\bar{k})$, and the inner product on $C_{G}\left(\bar{g}^{x w^{\prime}}\right) \cap C_{G}\left(\bar{h}^{w^{\prime}}\right) \leq C_{G}(\bar{k})$ is of $\theta_{\bar{k}}$-representations.

\section{Example: noncommutative fusion rules}

Noncommutative fusion rules for cocentral abelian extensions are not rare: choose $L=1, \sigma$ and $\tau$ trivial, and any nonabelian $G$, for instance. Also, see [Kosaki et al. 1997; Nikshych 1998; Zhu 2001]. We give here an example with $\sigma, \tau$ trivial, but nontrivial action of $L$. Let $G$ be the dihedral group of order 18, and let

$$
L \leq \text { Aut } D_{9} \cong \mathbb{Z}_{9} \rtimes \mathbb{Z}_{9}^{*} .
$$

Namely, $L=\langle 3\rangle \times\langle 4\rangle \cong \mathbb{Z}_{3} \times \mathbb{Z}_{3}$. [The first factor of $L$ is with respect to addition, the second, multiplication.] If we let $G=\left\langle x, y \mid x^{2}=y^{9}=e, y x=x y^{-1}\right\rangle$, then

$$
\left(x^{c} y^{d}\right)<(a, b):=x^{c} y^{a c+b d} \text {. }
$$

We choose $L$-orbit representatives $S=\left\{e, y^{3}, y^{6}, y, y^{2}, x, x y, x y^{2}\right\}$ with their respective stabilizers. Since $L$ is abelian, $\chi_{(g, \varphi)}^{(\ell)}=\chi_{(g, \varphi)}$ for all $\ell \in L$. Note that in the decomposition of the product of orbits, we have

$$
\mathcal{O}(x) \mathcal{O}(y)=3 \mathcal{O}(x y) \text { and } \mathcal{O}(y) \mathcal{O}(x)=3 \mathcal{O}\left(x y^{2}\right),
$$

which suffices to guarantee noncommutative fusion rules.

Theorem 6.1. Let $M(g, \alpha)$ denote the irreducible representation of $(\mathbb{F} G)^{*} \#(\mathbb{F} L)$ induced from $\alpha$, an irreducible representation of $L_{g}$ for $g \in S$. The first five rules are commutative.

i. $M(s, \alpha) \otimes M(t, \beta)=M(s t, \alpha \otimes \beta)$ for $s, t \in\left\langle y^{3}\right\rangle$.

ii. $M(s, \alpha) \otimes M(g, \beta)=M\left(g, \alpha \downarrow_{L_{g}} \otimes \beta\right)$ for $s \in\left\langle y^{3}\right\rangle, g \in\left\{y, y^{2}, x, x y, x y^{2}\right\}$.

iii. $M(g, \alpha) \otimes M(g, \beta)=3 M(h, \alpha \otimes \beta)$ if $\{g, h\}=\left\{y, y^{2}\right\}$.

$$
\text { iv. } M(y, \alpha) \otimes M\left(y^{2}, \beta\right)=\bigoplus_{s \in\left\langle y^{3}\right\rangle} \bigoplus_{\gamma \downarrow L_{y}=\alpha \otimes \beta} M(s, \gamma) \text {. }
$$$$
\text { v. } M(g, \alpha) \otimes M(g, \beta)=\bigoplus_{s \in\left\langle y^{3}\right\rangle} \bigoplus_{\gamma \downarrow_{L_{g}}=\alpha \otimes \beta} M(s, \gamma) \text { for } g \in\left\{x, x y, x y^{2}\right\} \text {. }
$$

The rest of the list holds for all $\alpha, \beta, \delta, \epsilon, \zeta, \eta, \mu, \nu$.

$$
\text { vi. } \begin{aligned}
M(y, \alpha) \otimes M(x, \beta) & =\bigoplus_{\text {all } \gamma} M\left(x y^{2}, \gamma\right)=M(x, \delta) \otimes M\left(y^{2}, \epsilon\right) \\
& =M\left(y^{2}, \zeta\right) \otimes M(x y, \eta)=M(x y, \mu) \otimes M(y, \nu) .
\end{aligned}
$$




$$
\text { vii. } \begin{aligned}
M(y, \alpha) \otimes M(x y, \beta) & =\bigoplus_{\text {all } \gamma} M(x, \gamma)=M(x y, \delta) \otimes M\left(y^{2}, \epsilon\right) \\
& =M\left(y^{2}, \zeta\right) \otimes M\left(x y^{2}, \eta\right)=M\left(x y^{2}, \mu\right) \otimes M(y, v) .
\end{aligned}
$$

$$
\text { viii. } \begin{aligned}
M(y, \alpha) \otimes M\left(x y^{2}, \beta\right) & =\bigoplus_{\text {all } \gamma} M(x y, \gamma)=M\left(x y^{2}, \delta\right) \otimes M\left(y^{2}, \epsilon\right) \\
& =M\left(y^{2}, \zeta\right) \otimes M(x, \eta)=M(x, \mu) \otimes M(y, \nu) .
\end{aligned}
$$

ix. $M(x, \alpha) \otimes M(x y, \beta)=\bigoplus_{\text {all } \gamma} M(y, \gamma)=M(x y, \delta) \otimes M\left(x y^{2}, \epsilon\right)$

$$
=M\left(x y^{2}, \zeta\right) \otimes M(x, \eta)
$$

x. $M(x, \alpha) \otimes M\left(x y^{2}, \beta\right)=\bigoplus_{\text {all } \gamma} M\left(y^{2}, \gamma\right)=M\left(x y^{2}, \delta\right) \otimes M(x y, \epsilon)$

$$
=M(x y, \zeta) \otimes M(x, \eta)
$$

Proof. Straightforward.

\section{Acknowledgement}

The author acknowledges the gracious comments of the referee, which led to major improvements in the paper.

\section{References}

[Andruskiewitsch 1996] N. Andruskiewitsch, "Notes on extensions of Hopf algebras", Canad. J. Math. 48:1 (1996), 3-42. MR 97c:16046 Zbl 0857.16033

[Andruskiewitsch and Natale 2003] N. Andruskiewitsch and S. Natale, "Braided Hopf algebras arising from matched pairs of groups", J. Pure Appl. Algebra 182 (2003), 119-149. MR 2004d:16064 Zbl 1024.16018

[Costache 2009] T.-L. Costache, "On irreducible projective representations of finite groups", Surv. Math. Appl. 4 (2009), 191-214. MR 2011h:20022 Zbl 1202.20015

[Dijkgraaf et al. 1991] R. Dijkgraaf, V. Pasquier, and P. Roche, "Quasi Hopf algebras, group cohomology and orbifold models", pp. 60-72 in Proceedings of the fourth meeting on theoretical physics: Recent advances in field theory (Annecy-le-Vieux, 1990), edited by P. Binétruy et al., Nuclear Phys. B Proc. Suppl. 18B, Elsevier, Amsterdam, 1991. MR 92m:81238 Zbl 0957.81670

[Etingof et al. 2005] P. Etingof, D. Nikshych, and V. Ostrik, "On fusion categories”, Ann. of Math. (2) 162:2 (2005), 581-642. MR 2006m:16051 Zbl 1125.16025

[Goff and Mason 2010] C. Goff and G. Mason, "Generalized twisted quantum doubles and the McKay correspondence”, J. Algebra 324:11 (2010), 3007-3016. MR 2011i:16046 Zbl 1228.16029

[Kashina et al. 2002] Y. Kashina, G. Mason, and S. Montgomery, "Computing the Frobenius-Schur indicator for Abelian extensions of Hopf algebras”, J. Algebra 251 (2002), 888-913. MR 2003f: 16061 Zbl 1012.16040 
[Kosaki et al. 1997] H. Kosaki, A. Munemasa, and S. Yamagami, "On fusion algebras associated to finite group actions”, Pacific J. Math. 177:2 (1997), 269-290. MR 98i:46064 Zbl 0882.46030

[Masuoka 2002] A. Masuoka, "Hopf algebra extensions and cohomology", pp. 167-209 in New directions in Hopf algebras, edited by S. Montgomery and H.-J. Schneider, Math. Sci. Res. Inst. Publ. 43, Cambridge Univ. Press, 2002. MR 2003d:16050 Zbl 1011.16024

[Montgomery 1993] S. Montgomery, Hopf algebras and their actions on rings, CBMS Regional Conference Series in Mathematics 82, American Mathematical Society, Providence, RI, 1993. MR 94i:16019 Zbl 0793.16029

[Natale 2003] S. Natale, "On group theoretical Hopf algebras and exact factorizations of finite groups", J. Algebra 270:1 (2003), 199-211. MR 2004k:16102 Zbl 1040.16027

[Natale 2005] S. Natale, "Frobenius-Schur indicators for a class of fusion categories", Pacific J. Math. 221:2 (2005), 353-377. MR 2007j:16070 Zbl 1108.16035

[Nauwelaerts and Van Oystaeyen 1991] E. Nauwelaerts and F. Van Oystaeyen, "Module characters and projective representations of finite groups", Proc. London Math. Soc. (3) 62:1 (1991), 151-166. MR 92f:20015 Zbl 0810.20012

[Nikshych 1998] D. Nikshych, " $K_{0}$-rings and twisting of finite-dimensional semisimple Hopf algebras”, Comm. Algebra 26:1 (1998), 321-342. MR 99d:16045a Zbl 0912.16018

[Schauenburg 2002] P. Schauenburg, "Hopf bimodules, coquasibialgebras, and an exact sequence of Kac”, Adv. Math. 165:2 (2002), 194-263. MR 2003e:16052 Zbl 1006.16054

[Thévenaz 1995] J. Thévenaz, G-algebras and modular representation theory, Oxford University Press, New York, 1995. MR 96j:20017 Zbl 0837.20015

[Witherspoon 2004] S. J. Witherspoon, "Products in Hochschild cohomology and Grothendieck rings of group crossed products", Adv. Math. 185:1 (2004), 136-158. MR 2005j:16010 Zbl 1063. 16012

[Zhu 2001] Y. Zhu, "Hecke algebras and representation ring of Hopf algebras", pp. 219-227 in First international congress of Chinese mathematicians (Beijing, 1998), AMS/IP Stud. Adv. Math. 20, Amer. Math. Soc., Providence, RI, 2001. MR 2002c:20011 Zbl 1064.20011

Communicated by Susan Montgomery

Received 2010-08-19 Revised 2011-02-28 Accepted 2011-04-10

cgoff@pacific.edu

Mathematics Department, University of the Pacific, 3601 Pacific Avenue, Stockton, CA 95211, United States 


\section{Algebra \& Number Theory}

msp.berkeley.edu/ant

\section{EDITORS}

MANAGING EDITOR

Bjorn Poonen

Massachusetts Institute of Technology

Cambridge, USA

\author{
EDITORIAL BOARD CHAIR \\ David Eisenbud \\ University of California \\ Berkeley, USA
}

\section{BOARD OF EDITORS}

Georgia Benkart

Dave Benson

Richard E. Borcherds

John H. Coates

J-L. Colliot-Thélène

Brian D. Conrad

Hélène Esnault

Hubert Flenner

Edward Frenkel

Andrew Granville

Joseph Gubeladze

Ehud Hrushovski

Craig Huneke

Mikhail Kapranov

Yujiro Kawamata

János Kollár

Yuri Manin

Barry Mazur

Philippe Michel

Susan Montgomery
University of Wisconsin, Madison, USA

University of Aberdeen, Scotland

University of California, Berkeley, USA

University of Cambridge, UK

CNRS, Université Paris-Sud, France

University of Michigan, USA

Universität Duisburg-Essen, Germany

Ruhr-Universität, Germany

University of California, Berkeley, USA

Université de Montréal, Canada

San Francisco State University, USA

Hebrew University, Israel

University of Kansas, USA

Yale University, USA

University of Tokyo, Japan

Princeton University, USA

Northwestern University, USA

Harvard University, USA

École Polytechnique Fédérale de Lausanne

University of Southern California, USA
Shigefumi Mori

Raman Parimala

Jonathan Pila

Victor Reiner

Karl Rubin

Peter Sarnak

Joseph H. Silverman

Michael Singer

Ronald Solomon

Vasudevan Srinivas

J. Toby Stafford

Bernd Sturmfels

Richard Taylor

Ravi Vakil

Michel van den Bergh

Marie-France Vignéras

Kei-Ichi Watanabe

Andrei Zelevinsky

Efim Zelmanov
RIMS, Kyoto University, Japan

Emory University, USA

University of Oxford, UK

University of Minnesota, USA

University of California, Irvine, USA

Princeton University, USA

Brown University, USA

North Carolina State University, USA

Ohio State University, USA

Tata Inst. of Fund. Research, India

University of Michigan, USA

University of California, Berkeley, USA

Harvard University, USA

Stanford University, USA

Hasselt University, Belgium

Université Paris VII, France

Nihon University, Japan

Northeastern University, USA

University of California, San Diego, USA

\section{PRODUCTION}

contact@msp.org

Silvio Levy, Scientific Editor

See inside back cover or www.jant.org for submission instructions.

The subscription price for 2012 is US \$175/year for the electronic version, and \$275/year (+\$40 shipping outside the US) for print and electronic. Subscriptions, requests for back issues from the last three years and changes of subscribers address should be sent to Mathematical Sciences Publishers, Department of Mathematics, University of California, Berkeley, CA 94720-3840, USA.

Algebra \& Number Theory (ISSN 1937-0652) at Mathematical Sciences Publishers, Department of Mathematics, University of California, Berkeley, CA 94720-3840 is published continuously online. Periodical rate postage paid at Berkeley, CA 94704, and additional mailing offices.

ANT peer review and production are managed by EditFLOW ${ }^{\circledR}$ from Mathematical Sciences Publishers.

PUBLISHED BY

mathematical sciences publishers

http://msp.org/

A NON-PROFIT CORPORATION

Typeset in IAT $_{\mathrm{E}} \mathrm{X}$

Copyright (C2012 by Mathematical Sciences Publishers 


\section{Algebra \& Number Theory}

Volume $6 \quad$ No. $2 \quad 2012$

Arithmetic of singular Enriques surfaces

Klaus HuleK and MatThias SchÜtT

An upper bound on the Abbes-Saito filtration for finite flat group schemes and applications

YICHAO TIAN

On the smallest number of generators and the probability of generating an algebra

Rostyslav V. Kravchenko, Marcin Mazur and Bogdan V. Petrenko

Moving lemma for additive higher Chow groups

AMALENDU KRISHNA and JINHYUN PARK

CHRISTOPHER GOFF

Uniformly rigid spaces

CHRISTIAN KAPPEN

On a conjecture of Kontsevich and Soibelman

LÊ QUY THUONG 